\title{
MedChemComm
}

Broadening the field of opportunity for medicinal chemists

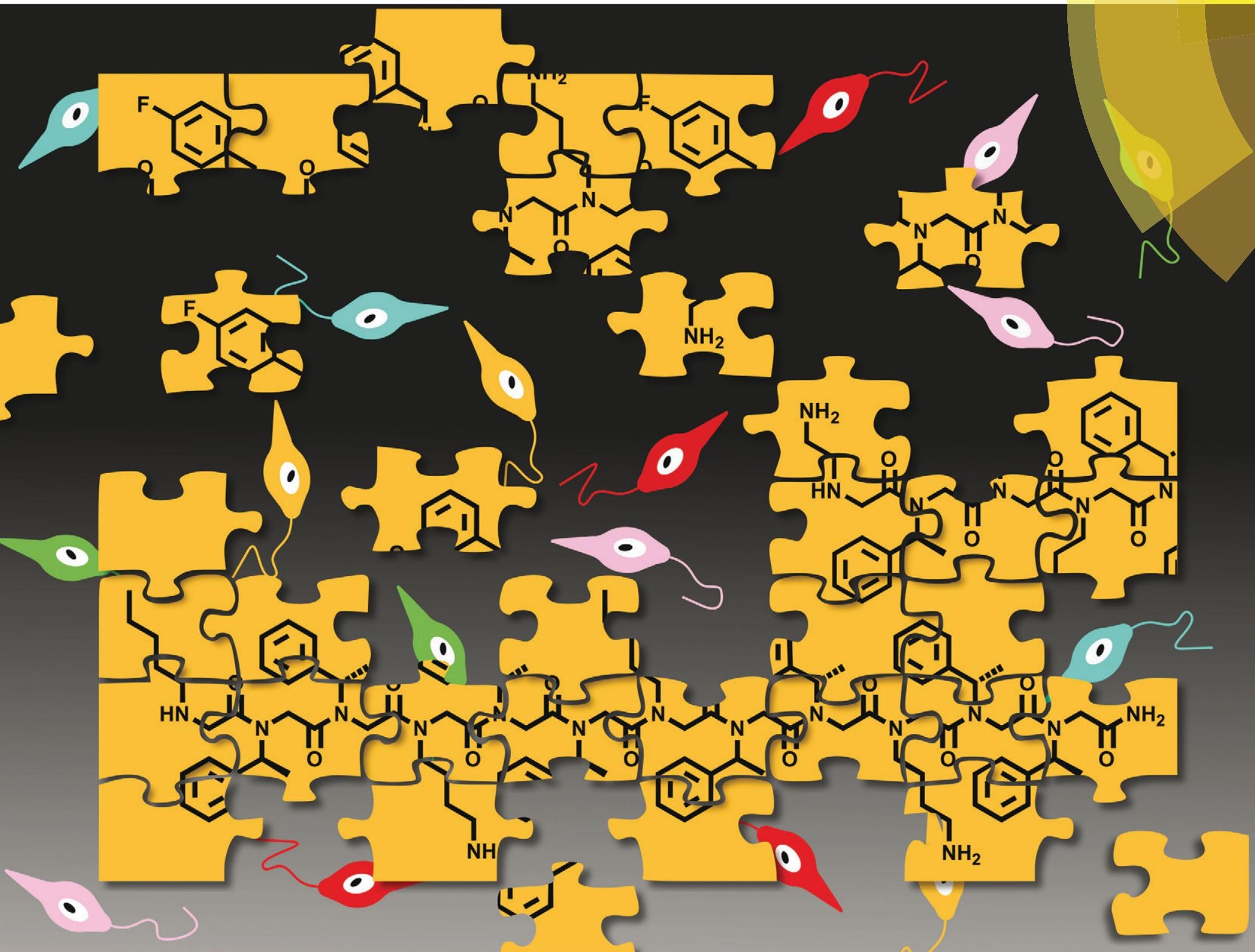

ISSN 2040-2503 relationship study of peptoids against Leishmania mexicana, a causative agent of cutaneous leishmaniasis

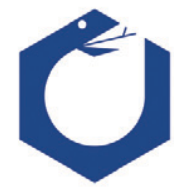

EFMC

European Federation for Medicinal Chemistry 


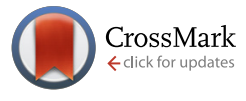

Cite this: Med. Chem. Commun., 2016, 7, 799

Received 27th January 2016, Accepted 10th March 2016

DOI: $10.1039 / c 6 m d 00060 f$

www.rsc.org/medchemcomm

\section{Enlarging the chemical space of anti-leishmanials: a structure-activity relationship study of peptoids against Leishmania mexicana, a causative agent of cutaneous leishmaniasis + t}

\author{
H. L. Bolt, $\S^{a}$ G. A. Eggimann, $\S^{a}$ Paul W. Denny ${ }^{\star a b}$ and Steven L. Cobb*a
}

\begin{abstract}
Peptoids, a class of peptide mimetics, have emerged as promising anti-infective agents against a range of bacterial and fungal infections. Recently we have shown peptoids to be novel anti-parasitic and, specifically, anti-leishmanial, compounds. In this study, we have expanded the chemical space of our peptoid library and have identified peptoids with low micromolar activity against Leishmania mexicana axenic amastigotes and significantly, the first peptoids with promising activity against intracellular amastigotes, which are the clinical cause of cutaneous leishmaniasis.
\end{abstract}

Leishmaniasis is a neglected tropical disease caused by insect vector borne protozoan parasites and is endemic in over 80 countries worldwide. It is estimated that more than 12 million people are currently infected and over 350 million people live at risk of infection. The vast majority of suffers live in areas of poverty where access to health care is often severely limited. Distinct Leishmania species can cause different forms of the disease; cutaneous leishmaniasis (CL), which leads to significant scaring and mucosal damage (mucocutaneous leishmaniasis), or visceral leishmaniasis (VL) causing life-threatening organ damage. ${ }^{1,2}$

At present, there is no vaccine available for the prevention of CL and VL, and current treatments rely on a limited selection of drugs, such as sodium stibogluconate (Pentostam) and meglumine antimoniate (Glucantime). These antimonials, requiring reduction to the trivalent $\mathrm{Sb}$ (III) form but with an unclear mode of action, have been in clinical use for over 70 years despite showing severe side-effects and requiring parenteral administration. ${ }^{3-5}$ Furthermore, the long usage of pentavalent antimonials in the treatment of leishmaniasis is leading to the emergence of drug resistance. Although resistance is not yet widespread, Leishmania spp. resistance to these drugs can be easily induced in a laboratory environment. ${ }^{4,5}$ Therefore second-line drugs such as amphotericin B

\footnotetext{
${ }^{a}$ Department of Chemistry, Biophysical Sciences Institute, Durham University, South Road, Durham, DH1 3LE, UK. E-mail: s.l.cobb@durham.ac.uk

${ }^{b}$ School of Medicine, Pharmacy and Health, Durham University, Queen's Campus, Stockton-on-Tees, TS17 6BH, UK. E-mail: p.w.denny@durham.ac.uk

$\dagger$ The authors declare no competing interests.

\$ Electronic supplementary information (ESI) available. See DOI: 10.1039/ c6md00060f

$\S$ These authors contributed equally to the project.
}

(Fungizone, a polyene antibiotic which binds parasite specific sterols) and the aromatic diamidine pentamidine (mechanism of action unclear) have been used increasingly. ${ }^{4,5}$ Both have been in clinical use for over 30 years, share the severe side-effects of antimonials and also require parenteral administration. The newest and only oral drug for VL, miltefosine (a phospholipid, originally developed as an anticancer drug, with an unverified mode of action against Leishmania spp.), is limited by its teratogenicity. ${ }^{4,5}$ Parasitic resistance towards these alternative drugs has not yet been conclusively confirmed in the field, but there are indications that it may only be a matter of time. ${ }^{4,6}$ Given the issues surrounding the use of the current first- and second-line drugs to treat leishmaniasis, there is clearly an urgent need to develop new and effective therapies.

Recently, there has been considerable interest in the discovery and development of safer, more effective treatments for VL. In the 2012 London Declaration on neglected tropical diseases, public and private partnerships pledged to help control VL by $2020 .{ }^{7}$ In contrast CL, a disease that causes 1.2 million new cases each year, remains relatively ignored. Although CL is usually non-fatal, it results in ulcerated lesions that can cause severe disfigurement and lead to other significant medical problems, disability and social exclusion.

Antimicrobial peptides (AMPs) have been proposed as one potential solution to the development of new topical agents to treat $\mathrm{CL}^{8-11}$ However, their inherent chemical and biological instability presents a major hurdle and only a few AMPs are currently in clinical trials as anti-bacterials. ${ }^{12}$

Peptoids, a class of peptide-mimetics, offer a better opportunity for the development of new topical anti-leishmanial therapeutics; they are cheaper to manufacture than peptide 
based drugs and are much more stable under physiological conditions with an improved biological lifetime. The in vitro activity of certain peptoids against both Gram-positive and Gram-negative bacteria and other clinically relevant targets are very similar to those of the leading AMPs. In addition, peptoids display the hallmark broad-spectrum activity of AMPs, suggesting a strong mechanistic similarity. ${ }^{13-17}$ We have recently shown that peptoids (or poly- $N$-substituted glycines) have potential as new anti-parasitic compounds against Leishmania mexicana, the causative agent of cutaneous leishmaniasis. ${ }^{18}$ In this study, we identified several peptoids with low micromolar activities, with the best 'hit' having an $\mathrm{ED}_{50}$ of $17 \mu \mathrm{M}$ against axenic L. mexicana the clinically relevant, mammalian stage of the parasite.

Herein, we have expanded our peptoid library to undertake a more detailed structure-activity relationship (SAR) study. Over 30 novel peptoids were synthesised and tested against L. mexicana promastigotes (insect stage parasites) and axenic amastigotes (mammalian stage parasites). Some peptoids from this library showed improved activity compared to our first library. The most promising peptoid compounds were also successfully screened, for the first time, against intracellular amastigotes (an in vitro model of disease) and their cytotoxicity investigated. Our results provide further evidence that peptoids may be a promising new class of anti-infectives, particularly in the search for improved therapeutics for the treatment of cutaneous leishmanaisis. These peptoids are both more stable to enzymatic degradation than previously analysed antimicrobial peptides, and show more promising activity against the clinically relevant amastigotes. $^{8}$

\section{Results and discussion}

In this extended SAR study, over 30 novel compounds were synthesised to further investigate the biological activity profile of peptoids against L. mexicana. The mode of action of peptoids is suspected to be membrane disruption so to add diversity to this library the sequences were designed around four motifs (see Fig. 1) with varying side chain substituents. We chose to add a range of substituted aromatic monomers to provide variation compared to our previous library ${ }^{18}$ and selected those derived from commercially available amines.

In motif 1 , peptoids include the subunit $N x N y N y$, which was repeated two, three or four times to give 6,9 or 12 residue peptoids respectively. For $N y$ different aromatic building blocks were chosen (see Fig. 1 for the structure of the side

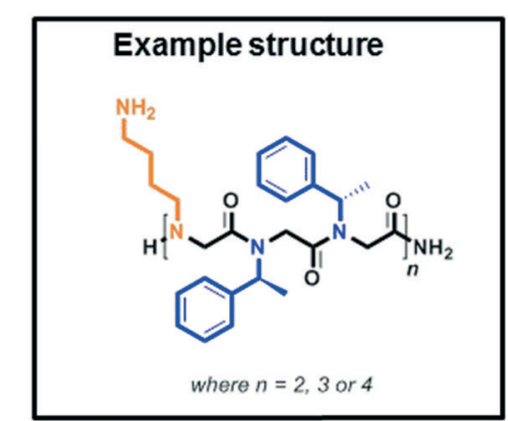

Motif 1
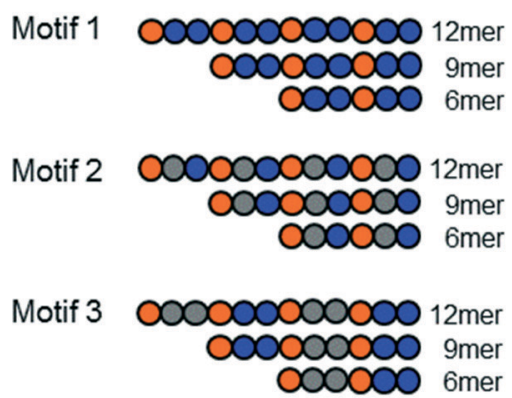

Motif 4

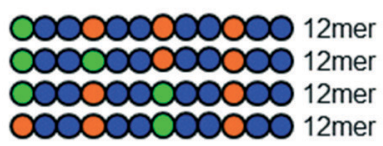

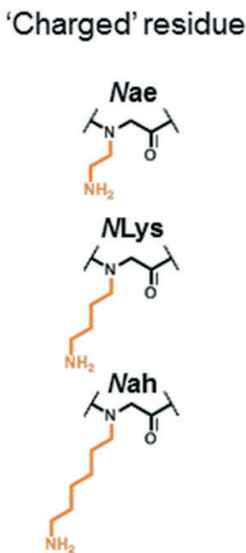

Aromatic residues

Alkyl residues

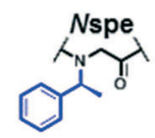

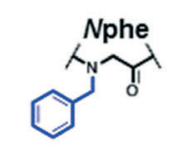<smiles>CC(C)C(=O)N(Cc1ccc(F)cc1)NC1CCCCC1</smiles>

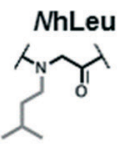

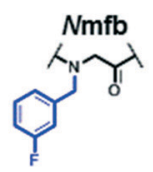<smiles>CCCCCN(CCCC)C(=O)CCC</smiles>

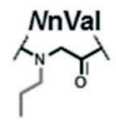

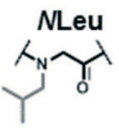

Namy
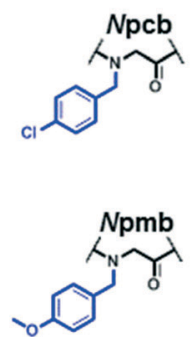

Fig. 1 An example of the peptoids synthesised and the chemical structures of subunits used in this peptoid library. The different structural motifs are shown to illustrate the repeating subunits in our sequences. 
chains). For the positively charged building block $N x$, lysinetype amines with different side chain lengths were chosen to study their influence on anti-leishmanial activity.

In a further iteration, as shown in Fig. 1 with motif 2 $(N \mathrm{x} N \mathrm{y} \mathrm{Nz})$, two different $\mathrm{Ny} / \mathrm{Nz}$ building blocks were used within the sequence. These residues were uncharged and either aromatic or alkyl amines. To investigate if the sequence specific position of $\mathrm{Ny} / \mathrm{Nz}$ with their different side chains influenced the activity, they were also placed in a co-block manner (motif 3). In a final design (motif 4), the overall positive charge of a 12 mer peptoid was reduced by replacing either one or two charged NLys residues with the uncharged Namy. Namy has the same molecular weight to NLys so differs only in the chemical functionality.

The peptoid library was synthesized manually on resin with the submonomer method using a shaker $\left(50{ }^{\circ} \mathrm{C}\right)$ and 15 min for each coupling or displacement step. All peptoids were purified by RP-HPLC to obtain a library of 49 compounds (for full details regarding synthesis and characterization, please see the ESI + . All peptoids were screened for activity against $L$. mexicana promastigotes and amastigotes. These results are shown as $\mathrm{ED}_{50}$ values in Table 1.

After having found active peptoids against the $L$. mexicana parasite in an earlier library, ${ }^{18}$ other aromatic building blocks were included within our motif of $N$ Lys $N y N y$ (Table 1). Using the building block Npmb in the 12 mer peptoid 19 , the activity against promastigotes reduced significantly by 3 fold $\left(\mathrm{ED}_{50}=42 \mu \mathrm{M}\right)$ compared to analogues containing Nphe (peptoid 4, $\mathrm{ED}_{50}=15 \mu \mathrm{M}$ ) or Nspe (peptoid 13, $\mathrm{ED}_{50}=8 \mu \mathrm{M}$ ). The shorter 6- and 9mer versions (peptoid 21 and 20 respectively) did not show any activity against the promastigotes and no activity could be found against amastigotes for any length of the Npmb peptoids.

In contrast, peptoids with chlorinated and fluorinated aromatic building blocks ( $N$ pcb, $N p f b$ and $N m f b ;$ peptoids 22-30) showed enhanced activity against the axenic amastigotes, whilst maintaining activity against promastigotes, the insect form of the parasite. Peptoids 22-24 have a chloro substituent in the para position of the phenyl ring ( $N p c b)$. These were all active against the promastigotes, but only the longer 9- and 12mer (peptoids 22 and 23) also showed any activity against the axenic amastigotes with $\mathrm{ED}_{50}$ values of 85 or $44 \mu \mathrm{M}$, respectively. In case of the peptoids with fluorine atoms in the para or meta positions, only the longest 12 residue peptoids showed modest toxicity against the amastigotes (peptoid 25: $\mathrm{ED}_{50}=75 \mu \mathrm{M}$ and peptoid 28: $\mathrm{ED}_{50}=69 \mu \mathrm{M}$ ). However, fluorinated peptoids had a good efficiency against promastigotes, with similarly low $\mathrm{ED}_{50}$ values as the nonhalogenated analogues. Even the shorter 9 residue peptoids, 26 and 29, showed anti-parasitic activity against promastigotes in the low $\mu \mathrm{M}$ range. The short 6mers (peptoid 27 and 30) did not show any activity against either stage of the parasite.

Our previous study ${ }^{18}$ showed an increase in activity when the chiral Nspe residue was included within the sequence compared to Nphe. Therefore, the chiral aromatic building block Nspe was then combined with the fluorinated building block $N$ pfb in either motif 2 or 3 (peptoids 31-36). Interestingly, the analogues of both motifs had similar activity against both promastigotes and axenic amastigotes. The longest 12 residue peptoids, 31 and 34, showed very good activity against promastigotes $\left(\mathrm{ED}_{50}\right.$ values of 8 and $\left.6 \mu \mathrm{M}\right)$. This activity was similar to the Nspe-only template peptoid $13\left(\mathrm{ED}_{50}=\right.$ $8 \mu \mathrm{M}$ ) and about 2 fold better compared to the Npfb-only peptoid $25\left(\mathrm{ED}_{50}=15 \mu \mathrm{M}\right)$. However, the difference in activity between 31/34 and 13 was very different against the axenic amastigote form; the Nspe- $N$ pfb-mixed peptoids 31 and 34 showed good activity $\left(\mathrm{ED}_{50}\right.$ values of 27 and $21 \mu \mathrm{M}$ respectively) whereas the Nspe-only peptoid 13 was completely inactive and the $\mathrm{Npfb}$-only peptoid 25 showed only moderate activity with an $\mathrm{ED}_{50}$ of $75 \mu \mathrm{M}$. Therefore, the combination of two building blocks with different activities against promastigotes and axenic amastigotes can help to improve the overall activity against the parasite. Peptoid 34 was the most active compound from this part of the SAR study with $\mathrm{ED}_{50}$ values of $6 \mu \mathrm{M}$ (promastigotes) and $21 \mu \mathrm{M}$ (axenic amastigotes).

The library was then extended to include sequences that combine one aromatic residue and one residue with an alkyl side chain within the same peptoid. These peptoids were based on motif 2 and the monomers NnVal, NLeu and NhLeu were used to synthesize peptoids 37-45. As shown in Table 1, none of these peptoids showed much activity against either lifecycle stage of $L$. mexicana. Only the 12 and 9 residue peptoids containing the NhLeu building block were active against the promastigote form, (peptoid $43 \mathrm{ED}_{50}=12 \mu \mathrm{M}$, peptoid $44 \mathrm{ED}_{50}=52 \mu \mathrm{M}$ ). These results highlight that the size and chemical functionality of residues in the motif $N \mathrm{x} N y N z$ is important for anti-leishmanial efficacy.

Our previous study showed that the hydrophobicity of side chains in the peptoid sequence influenced the anti-parasitic activity of the compound; the shorter Nae residue significantly increased the biological activity against the amastigote form of the parasite compared to the NLys building block. ${ }^{18}$ To extend the SAR study, NLys residues were replaced by the alkyl building block Namy in several sequences. In these peptoids, the terminal $-\mathrm{NH}_{2}$ group of one or two NLys side chains is replaced by a $-\mathrm{CH}_{3}$ group. Thus, the net positive charge of the peptoid is reduced, but has the same molecular weight as the NLys analogues, as shown in Table 1 (peptoids 46-49).

For most compounds in this library (peptoids 1-45), the promastigotes are more susceptible to the peptoids than amastigotes. We believe this is due to the large differences in the cell surface coat of each parasite. ${ }^{19}$ However, surprisingly, all four Namy peptoids (46-49) showed very similar activity not only against the promastigotes, but also against the amastigotes. For the promastigotes, $\mathrm{ED}_{50}$ values were between 8-10 $\mu \mathrm{M}$ and therefore comparable to our previous 'hit', peptoid $16\left(\mathrm{ED}_{50}=7 \mu \mathrm{M}\right)$. The activity against the amastigotes was also in the low $\mu \mathrm{M}$ range with $\mathrm{ED}_{50}$ values between 15-21 $\mu \mathrm{M}$ and similar to peptoid $16\left(\mathrm{ED}_{50}=17 \mu \mathrm{M}\right)$. 
Table 1 Chemical and biological data for peptoids. For side chain abbreviations see Fig. 1. All peptoids are amidated at the C-terminus

\begin{tabular}{|c|c|c|c|}
\hline \multirow[b]{2}{*}{ Peptoid } & \multirow[b]{2}{*}{ Sequence } & \multicolumn{2}{|l|}{$\mathrm{ED}_{50}(\mu \mathrm{M})$} \\
\hline & & Promastigotes & Amastigotes \\
\hline $\mathbf{1}^{a}$ & $(N a h N p h e N p h e)_{4}$ & 21 & $>100$ \\
\hline $2^{a}$ & $(N a h N p h e N p h e)_{3}$ & $>100$ & $>100$ \\
\hline $3^{a}$ & $(\mathrm{NahNpheNphe})_{2}$ & $>100$ & $>100$ \\
\hline $4^{a}$ & $(N L y s N p h e N p h e)_{4}$ & 15 & $>100$ \\
\hline $5^{a}$ & $(N \text { Lys } N \text { phe } N p h e)_{3}$ & $>100$ & $>100$ \\
\hline $6^{a}$ & $(N L y s N p h e N p h e)_{2}$ & $>100$ & $>100$ \\
\hline $7^{a}$ & $(N a e N p h e N p h e)_{4}$ & 21 & $>100$ \\
\hline $8^{a}$ & $(N a e N p h e N p h e)_{3}$ & $>100$ & $>100$ \\
\hline $9^{a}$ & $(\mathrm{NaeNpheNphe})_{2}$ & $>100$ & $>100$ \\
\hline $10^{a}$ & $(\mathrm{NahNspeNspe})_{4}$ & 11 & $>100$ \\
\hline $11^{a}$ & $(\mathrm{NahNspeNspe})_{3}$ & 25 & $>100$ \\
\hline $12^{a}$ & $(N a h N s p e N s p e)_{2}$ & $>100$ & $>100$ \\
\hline $13^{a}$ & $(N L y s N \text { speNspe })_{4}$ & 8 & $>100$ \\
\hline $14^{a}$ & $(\text { NLysNspeNspe })_{3}$ & 15 & $>100$ \\
\hline $15^{a}$ & $(\mathrm{NLysNspeNspe})_{2}$ & $>100$ & $>100$ \\
\hline $16^{a}$ & $(\mathrm{NaeNspeNspe})_{4}$ & 7 & 17 \\
\hline $17^{a}$ & $(\mathrm{NaeNspeNspe})_{3}$ & 10 & $>100$ \\
\hline $18^{a}$ & $(\mathrm{NaeNspeNspe})_{2}$ & $>100$ & $>100$ \\
\hline 19 & $(N L y s N p m b N p m b)_{4}$ & 42 & $>100$ \\
\hline 20 & $(N L y s N p m b N p m b)_{3}$ & $>100$ & $>100$ \\
\hline 21 & $(N L y s N p m b N p m b)_{2}$ & $>100$ & $>100$ \\
\hline 22 & $(N L y s N p c b N p c b)_{4}$ & 28 & 44 \\
\hline 23 & $(N L y s N p c b N p c b)_{3}$ & 22 & 85 \\
\hline 24 & $(N L y s N p c b N p c b)_{2}$ & 29 & $>100$ \\
\hline 25 & $(N L y s N p f b N p f b)_{4}$ & 15 & 75 \\
\hline 26 & $(N L y s N p f b N p f b)_{3}$ & 19 & $>100$ \\
\hline 27 & $(N L y s N p f b N p f b)_{2}$ & $>100$ & $>100$ \\
\hline 28 & $(N \mathrm{Lys} N \mathrm{mfb} N \mathrm{Nmfb})_{4}$ & 14 & 69 \\
\hline 29 & $(N L y s N m f b N m f b)_{3}$ & 17 & $>100$ \\
\hline 30 & $(N \mathrm{Lys} N \mathrm{mfb} N \mathrm{Nmfb})_{2}$ & $>100$ & $>100$ \\
\hline 31 & $(N L y s N p f b N s p e)_{4}$ & 8 & 27 \\
\hline 32 & $(N L y s N p f b N s p e)_{3}$ & 13 & $>100$ \\
\hline 33 & $(N L y s N p f b N s p e)_{2}$ & $>100$ & $>100$ \\
\hline 34 & {$[(N L y s N p f b N p f b)(N L y s N \text { spe } N \text { spe })]_{2}$} & 6 & 21 \\
\hline 35 & $(N L y s N$ speNspe)(NLys $N$ pfbNpfb)(NLysNspeNspe) & 13 & $>100$ \\
\hline 36 & $(N L y s N p f b N p f b)(N L y s N s p e N s p e)$ & $>100$ & $>100$ \\
\hline 37 & $(N L y s N n V a l N s p e)_{4}$ & $>100$ & $>100$ \\
\hline 38 & $(N L y s N n V a l N s p e)_{3}$ & $>100$ & $>100$ \\
\hline 39 & $(N L y s N n V a l N s p e)_{2}$ & $>100$ & $>100$ \\
\hline 40 & $(N L y s N L e u N s p e)_{4}$ & $>100$ & $>100$ \\
\hline 41 & $(N L y s N L e u N s p e)_{3}$ & $>100$ & $>100$ \\
\hline 42 & $(N L y s N L e u N s p e)_{2}$ & $>100$ & $>100$ \\
\hline 43 & $(\text { NLysNhLeuNspe })_{4}$ & 12 & $>100$ \\
\hline 44 & $(\text { NLysNhLeuNspe })_{3}$ & 52 & $>100$ \\
\hline 45 & $(N L y s N h L e u N s p e)_{2}$ & $>100$ & $>100$ \\
\hline 46 & $($ NamyNspeNspe $)[(\text { NLysNspeNspe })]_{3}$ & 8 & 21 \\
\hline 47 & $(\text { NamyNspeNspe })_{2}(\text { NLysNspeNspe })_{2}$ & 11 & 16 \\
\hline 48 & {$[(\text { NamyNspeNspe })(N L y s N \text { speNspe })]_{2}$} & 10 & 17 \\
\hline 49 & $(N L y s N \text { spe } N \text { spe })_{2}($ NamyNspeNspe $)(N L y s N$ spe $N$ spe $)$ & 10 & 15 \\
\hline
\end{tabular}

These results suggest that the increased hydrophobicity and reduced charge of the Namy residues can increase biological activity compared to shorter alkyl substituents and it does not matter if the overall positive charge is reduced by 1 or 2 or which residue is replaced. ${ }^{19,20}$

All aforementioned results discuss assays against axenic, i.e. extracellular, cultured amastigotes. In the clinical stage of leishmaniasis the amastigotes reside within macrophages. Triage from these axenic assays highlighted four promising sequences from our library that were screened against $L$. mexicana infected RAW264.7 murine macrophages. The results were obtained using an assay system recently described for screening compounds against $L$. donovani infected cells, ${ }^{21-23}$ with some modifications to suit our cell lines, and are summarised in Table 2/Fig. 2 .

Peptoid 7 and 16 were chosen from our earlier study. Peptoid 16 (NaeNspeNspe) ${ }_{4}$ was the most potent peptoid from the previous library and showed low $\mu \mathrm{M}$ activity against both promastigote and axenic amastigote forms of the parasite. Peptoid 7 is an analogue of peptoid 16 and only showed 
Table 2 Biological data for intracellular amastigote assay. Compounds tested against, L. mexicana promastigotes, axenic amastigotes, intracellular amastigotes and also RAW264.7 macrophage cells. All peptoids are amidated at the C-terminus. For side chain abbreviations see Fig. 1

\begin{tabular}{|c|c|c|c|c|c|}
\hline Compound & Peptoid sequence & \multicolumn{4}{|l|}{$\mathrm{ED}_{50}(\mu \mathrm{M})$} \\
\hline Amphotericin B & & $<2$ & $<2$ & 0.195 & $>5$ \\
\hline 16 & $(\mathrm{NaeNspeNspe})_{4}$ & 7 & 17 & $>5$ & 3 \\
\hline 34 & {$[(N \text { Lys } N \text { pfb } N \text { pfb })(N \text { Lys } N \text { spe } N s p e)]_{2}$} & 6 & 21 & $>5$ & 5 \\
\hline 47 & $(\text { NamyNspeNspe })_{2}(\text { LlysNspeNspe })_{2}$ & 11 & 16 & 1.6 & 1.7 \\
\hline
\end{tabular}

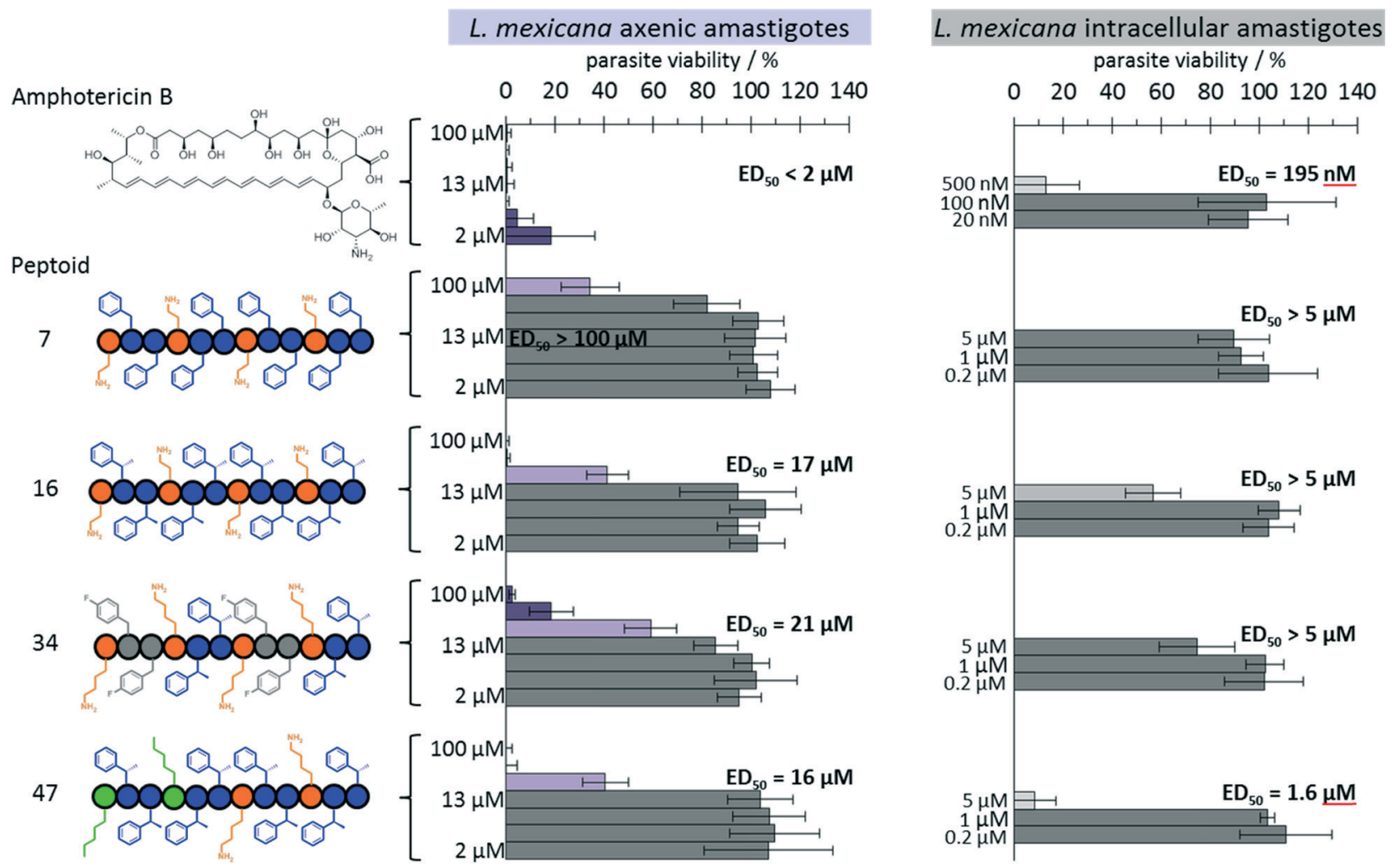

Fig. 2 Biological data for intracellular L. mexciana amastigote assay. Briefly, RAW 264.7 murine macrophages were subcultured in 96-well plates and incubated with L. mexicana amastigotes for 24 hours. Infected macrophages were treated with selected peptoids for 24 hours then lysed with SDS solution to release L. mexicana. Following a 48 hour incubation, parasite viability was assessed using the alamarBlue reagent. For cytotoxicity against macrophages only, the protocol was repeated in the absence of parasites.

activity against promastigotes so was used as negative control in our assay. From this study, peptoid 34 was chosen to investigate the mixed use of the aromatic building blocks Nspe and $N$ pfb. Peptoid 47, was chosen to examine the effect of a reduced overall positive charge and greater hydrophobicity of the Namy residue.

The results show that not all peptoids with activity against axenic amastigotes can retain their activity against the intracellular amastigotes. Encouragingly, peptoid 16 showed some activity against the intracellular parasites at $5 \mu \mathrm{M}$, the highest concentration measured, whilst peptoid 34 was only moderately active at this concentration. However, peptoid 47, which had the lowest $\mathrm{ED}_{50}$ value against axenic amastigotes (16 $\mu \mathrm{M})$, was also the most active against the intracellular amastigotes with an $\mathrm{ED}_{50}$ of $1.6 \mu \mathrm{M}$. Peptoid 7 was, as expected, non-active under the concentrations tested indicating that peptoids with little activity against the axenic amastigotes are likely to have poor activity against intramacrophage amastigotes.

However, it is noteworthy, and consistent with studies from other groups, ${ }^{16}$ that peptoids with increased antimicrobial activity also demonstrated increased host cell cytotoxicity (Table 2), limiting the range of concentrations studied in the infection assays to $\leq 5 \mu \mathrm{M}$. For example, peptoids 16 and 34 demonstrated greater toxicity against the RAW264.7 macrophages than the intracellular (and axenic) amastigotes $\left(\mathrm{ED}_{50}\right.$ $>5$ vs. the intracellular parasite against $\mathrm{ED}_{50}$ of $3 \mu \mathrm{M}$ and 5 $\mu \mathrm{M}$ respectively $v s$. the macrophages); and peptoid 47 is equivalently cytotoxic to both host and parasite $\left(\mathrm{ED}_{50}\right.$ of 1.7 and 1.6 respectively). Whilst further screening determine the 
toxicity of the complete library against the host cell may have some value for future consideration of the cytotoxicity and mode of action of peptoids, this study sought to establish the selective nature of peptoids identified as having significant potency against $L$. mexicana axenic amastigotes.

Clearly overcoming host cell cytotoxicity is a general problem for peptoids, however the fact that peptoid 47 demonstrated low micromolar activity against $L$. mexciana infected macrophages may provide a starting for studies of the antimicrobial and cytotoxic effects of peptoids. Despite the host cell toxicity the data gathered from the peptoid library (and specifically peptoids 16, 34 and 47) will help to further elucidate the key characteristics required for anti-leishmanial compound development.

\section{Conclusions}

In conclusion within this study we have significantly enlarged the peptoid activity data available for the development of potential anti-leishmanials. Peptoid 47 (NamyNspeNspe $)_{2}(N \text { LysNspeNspe })_{2}$ is the first peptoid published to have activity in the low $\mu \mathrm{M}$ range against $L$. mexicana promastigotes and axenic amastigotes, and also retain activity against the clinically significant intramacrophage amastigotes. Despite evident host macrophage cytotoxicity, 47 is a promising starting point for peptoidbased anti-leishmanials.

Since any potential treatments for cutaneous leishmaniasis could be formulated for topical application, a certain level of toxicity to host cells may be acceptable. To overcome or reduce the toxicity against macrophages future medicinal chemistry investigations will need to be undertaken, perhaps to maximise delivery of the peptoid to the parasite by optimising host cell penetration. Given that the predicted mode of action of peptoids is plasma membrane poreformation or disruption ${ }^{17}$ rather than inhibition of a protein target, the risk of development of parasite resistance is low, conferring an advantage to this approach in treatment.

In summary, the results described provide further evidence that peptoids are a promising new class of anti-infectives, particularly in the search for improved topical therapeutics for the treatment of CL.

\section{Acknowledgements}

We thank the Engineering and Physical Sciences Research Council (Durham University DTG, HLB) and the Swiss National Science Foundation (GAE) for financial support.

\section{Notes and references}

1 S. Y. Chon, H. Q. Doan, R. M. Mays, S. M. Singh, R. A. Gordon and S. K. Tyring, Antibiotic overuse and resistance in dermatology, Dermatol. Ther., 2012, 25, 55-69.

2 J. Alvar, I. D. Velez, C. Bern, M. Herrero, P. Desjeux, J. Cano, J. Jannin and M. den Boer, Leishmaniasis worldwide and global estimates of its incidence, PLoS One, 2012, 7, e35671.
3 L. Kedzierski, Leishmaniasis Vaccine: Where are We Today?, J. Global Infect. Dis., 2010, 2, 177-185.

4 S. L. Croft, S. Sundar and A. H. Fairlamb, Drug resistance in leishmaniasis, Clin. Microbiol. Rev., 2006, 19, 111-126.

5 N. Singh, M. Kumar and R. K. Singh, Leishmaniasis: current status of available drugs and new potential drug targets, Asian Pac. J. Trop. Med., 2012, 5, 485-4974.6.

6 M. Ephros, E. Waldman and D. Zilberstein, Pentostam induces resistance to antimony and the preservative chlorocresol in Leishmania donovani promastigotes and axenically grown amastigotes, Antimicrob. Agents Chemother., 1997, 41, 1064-1068.

7 London Declaration on Neglected Tropical Diseases, www.dndi. org/images/stories/press_kit/PressRoom/NTDs/NTD_Event_ London_Declaration.pdf.

8 F. L. Chadbourne, C. Raleigh, H. Z. Ali, P. W. Denny and S. L. Cobb, Studies on the antileishmanial properties of the antimicrobial peptides temporin A, B and 1Sa, J. Pept. Sci., 2011, 17, 751-755.

9 S. L. Cobb and P. W. Denny, Antimicrobial peptides for leishmaniasis, Curr. Opin. Invest. Drugs, 2010, 11, 868-875.

10 M. L. Mangoni, J. M. Saugar, M. Dellisanti, D. Barra, M. Simmaco and L. Rivas, Temporins, small antimicrobial peptides with leishmanicidal activity, J. Biol. Chem., 2005, 280, 984-990.

11 S. Dabirian, Y. Taslimi, F. Zahedifard, E. Gholami, F. Doustdari, M. Motamedirad, S. Khatami, K. Azadmanesh, S. Nylen and S. Rafati, Human Neutrophil Peptide-1 (HNP-1): A New Anti-Leishmanial Drug Candidate, PLoS Neglected Trop. Dis., 2013, 7, e2491.

12 D. K. Mercer and D. A. O'Neil, Peptides as the next generation of anti-infectives, Future Med. Chem., 2013, 5, 315-337.

13 M. T. Dohm, R. Kapoor and A. E. Barron, Peptoids: bioinspired polymers as potential pharmaceuticals, Curr. Pharm. Des., 2011, 17, 2732-2747.

14 M. L. Huang, M. A. Benson, S. B. Y. Shin, V. J. Torres and K. Kirshenbaum, Amphiphilic Cyclic Peptoids That Exhibit Antimicrobial Activity by Disrupting Staphylococcus aureus Membranes, Eur. J. Org. Chem., 2013, 3560-3566.

15 R. Kapoor, M. W. Wadman, M. T. Dohm, A. M. Czyzewski, A. M. Spormann and A. E. Barron, Antimicrobial peptoids are effective against Pseudomonas aeruginosa biofilms, Antimicrob. Agents Chemother., 2011, 55, 3054-3057.

16 B. Mojsoska, R. N. Zuckermann and H. Jenssen, Structureactivity relationship study of novel peptoids that mimic the structure of antimicrobial peptides, Antimicrob. Agents Chemother., 2015, 59, 4112-4120.

17 N. P. Chongsiriwatana, J. A. Patch, A. M. Czyzewski, M. T. Dohm, A. Ivankin, D. Gidalevitz, R. N. Zuckermann and A. E. Barron, Peptoids that mimic the structure, function, and mechanism of helical antimicrobial peptides, Proc. Natl. Acad. Sci. U. S. A., 2008, 105, 2794-2799.

18 G. A. Eggimann, H. L. Bolt, P. W. Denny and S. L. Cobb, Investigating the Anti-leishmanial Effects of Linear Peptoids, ChemMedChem, 2015, 10, 233-237. 
19 G. A. Eggimann, K. Sweeney, H. L. Bolt, N. Rozatian, S. L. Cobb and P. W. Denny, The Role of Phosphoglycans in the Susceptibility of Leishmania mexicana to the Temporin Family of Anti-Microbial Peptides, Molecules, 2015, 20, 2775-2785.

20 M. Torrent, D. Pulido, L. Rivas and D. Andreu, Antimicrobial peptide action on parasites, Curr. Drug Targets, 2012, 13, 1138-1147.

21 S. K. Jain, R. Sahu, L. A. Walker and B. L. Tekwani, A parasite rescue and transformation assay for antileishmanial screening against intracellular Leishmania donovani amastigotes in THP1 human acute monocytic leukemia cell line, J. Visualized Exp., 2012, 30, 4054.

22 D. Paape, A. S. Bell, W. P. Heal, J. A. Hutton, R. J. Leatherbarrow, E. W. Tate and D. F. Smith, Using a nonimage-based medium-throughput assay for screening compounds targeting $\mathrm{N}$-myristoylation in intracellular Leishmania amastigotes, PLoS Neglected Trop. Dis., 2014, 8, e3363.

23 P. A. Bates, Complete developmental cycle of Leishmania mexicana in axenic culture, Parasitology, 1994, 108, 1-9. 